\title{
Colocando a Saúde Materna no Centro das Políticas de Saúde Pública ${ }^{1}$
}

\section{Putting Maternal Health at the Centre of Public Health Policies}

\author{
Marianna Leite \\ Birkbeck College - University of London \\ mleite03@mail.bbk.ac.uk
}

\section{Resumo}

Traçando um processo de análise histórica e política, este artigo trata a extensão que os serviços de saúde reprodutiva têm sido marginalizados pelos processos de reforma no setor de saúde no Brasil ao longo das últimas três décadas. Esta marginalização teve implicações significativas, que se refletem no fracasso contínuo do país para minorar as altas taxas de mortalidade materna, apesar da constante ênfase colocada sobre essas metas, por exemplo através do Millennium Development Goals. Além disso, os discursos em torno da capacidade de uma abordagem baseada em direitos para cumprir estes objetivos através de estratégias como a descentralização, continuam a ignorar estruturas sociais subjacentes, que influenciam o poder de decisão e o sentido da noção de direitos.

Palavras-chave: Reformas do Setor da Saúde; Descentralização de Serviços; Mortalidade Materna; Brasil.

\begin{abstract}
Drawing on a process of historical and policy analysis, this article considers the extent to which reproductive health services have been marginalized by the reform processes in Brazil's health sector over the past three decades. This marginalization has had significant implications that are reflected in the country's continued failure to tackle high maternal mortality rates despite renewed emphasis placed on these goals, for example via the Millennium Development Goals. Moreover, discourses surrounding the capacity of a rights-based approach to fulfil these goals through strategies such as decentralisation continue to ignore underlying social structures that influence power, agency and context grounding the notion of rights.
\end{abstract}

Keywords: Health Sector Reforms; Decentralisation of Services; Maternal Mortality; Brazil. 


\section{Introdução}

Baseando-se em um processo de análise histórica e política, este artigo procurou determinar as razões dos serviços de saúde reprodutiva não se beneficiaram com as supostas vantagens da descentralização. $\mathrm{O}$ estudo de caso brasileiro está situado dentro de um contexto de debates mais amplos da reforma do setor de saúde na América Latina, as desigualdades de acesso aos cuidados de saúde e suas implicações na saúde das mulheres e a igualdade de gênero. Ele aborda como legados políticos vindos do exterior e a política interna do país podem ter influenciado para o uso de um discurso sobre os direitos reprodutivos, quando na verdade perpetuaram uma agenda neoliberal de controle populacional.

\section{O Programa Millennium Development Goal (MDG) e os Resultados de Saúde Materna}

O progresso global de realização da Meta 5 do programa Millennium Development Goal (MDG) de reduzir $75 \%$ da taxa de mortalidade materna de 1990 até 2015 é crucial para a integração efetiva das perspectivas de gênero para políticas de saúde e acompanhamento (VICTORA et al, 2011). É amplamente estabelecido de que o gênero é um fator importante de vulnerabilidade social e econômica, que se manifesta em desigualdades no acesso aos cuidados de saúde (STANDING, 1997). No entanto, segundo estimativas de 2008, mais de 350.000 mulheres ainda morrem anualmente de complicações tratáveis ou evitáveis da gravidez e do parto por ano (WHO et al, 2010). Um impressionante total de $99 \%$ destas mortes ocorre nos países em desenvolvimento (WHO et al, 2010).

A importância desses números não pode ser subestimada. O Relatório de Desenvolvimento Humano de 2010 afirma que os dados de mortalidade materna são um aspecto crucial de bem-estar (PNUD, 2010). Bem-estar no sentido de sobrevivência, de alguém levar uma vida longa e saudável, de estar bem nutrido, poder 'viver decentemente' e poder realizar seus projetos de vida (SEN, 2002; ALKIRE, 2005). É um indicador de status na sociedade e parte dos direitos fundamentais de uma pessoa poder viver a vida com dignidade e de acordo com suas limitações e capacidades (NUSSBAUM, 2001). Portanto, obter informações sobre as disparidades de gênero nas políticas de saúde pode ajudar a superar as desvantagens crônicas de alguns grupos no seu acesso ao bem-estar e capacitação de gerir sua própria vida
(NUSSBAUM, 2001).

O Relatório de Desenvolvimento Humano de 2010 indica que os sistemas nacionais de saúde reprodutiva da América Latina e do Caribe são um dos maiores contribuintes para a desigualdade de gênero no mundo (PNUD, 2010). O Brasil é relatado como o paradoxo mais flagrante da região (UNDP, 2010; WHO, 2004). A literatura sobre o assunto sugere que, apesar da introdução de medidas reguladoras para aumentar a eficiência e reduzir as desigualdades no setor da saúde, o acesso à saúde permanece extremamente desigual nesse país (VICTORA et al, 2011). O Sistema único de Saúde do Brasil deveria garantir igualdade de acesso e qualidade dos serviços a todos (VICTORIA et al, 2011). No entanto, as pessoas de grupos vulneráveis e de baixa renda tem mais dificuldade em obter acesso a serviços de saúde de qualidade e as mulheres ainda são mais desproporcionalmente afetadas (VICTORA et al, 2011; ALMEIDA et al, 2000).

O Brasil tem feito progressos significativos para enfrentar os desafios em outras áreas do setor da saúde e alguns indicadores melhoraram muito, com o país igualando padrões internacionalmente aceitos como satisfatórios (VICTORA et al, 2011). Em especial, programas como imunizações, HIV/AIDS e a mortalidade infantil tem tido relativo sucesso (WHO, 2004). No entanto, apesar do crescimento econômico do Brasil, os indicadores de saúde reprodutiva permaneceram ruins (VICTORA et al, 2011). Enquanto globalmente o número de mulheres que morrem devido a complicações relacionadas à gravidez e o parto diminuiu 34\% entre 1990 e 2008 (WHO et al, 2010), no Brasil essa redução foi de apenas 3,9\% (HOGAN et al, 2010).

\section{Abordagens Baseadas em Direitose Saúde Materna}

O uso da linguagem dos direitos tem crescido rapidamente na formulação das políticas e em sua aplicação (CORNWALL \& MOLYNEUX, 2006). No entanto, os direitos formais, como preconizados por organismos internacionais de desenvolvimento, nem sempre bastam para melhorar a realidade cotidiana das mulheres (CORNWALL \& MOLYNEUX, 2006; CORNWALL \& NYAMU-MUSEMBI, 2004; VAUGHAN, 2010). Modelos trazidos de fora, baseados na importância dos direitos, muitas vezes ignoraram o complexo contexto local que restringe o cumprimento do dever Estatal e os direitos dos cidadãos (CORNWALL \& NYAMU-MUSEMBI, 2004).

Além disso, as estruturas de desigualdade de 
gênero dentro da sociedade condicionam profundamente as atitudes em relação ao discurso baseado em direitos, e as iniciativas a ele relacionadas (UNTERHALTER, 2003). A persistência da injustiça de gênero, associada com identificação entre os papéis masculino e feminino nas relações de casal e com os demais membros da família, tornam as perspectivas de mudança particularmente difíceis (GREANY, 2008; UNTERHALTER, 2010).

A implantação de uma política de melhores serviços de saúde é ferrenhamente combatida pela ala conservadora da Igreja Católica, que contesta todos os avanços na área de direitos sexuais e reprodutivos (DOYAL, 1995; CORRÊA, 2010). E com o aumento do pluralismo religioso na América Latina, a oposição aos direitos sexuais e reprodutivos abre outras frentes, incluindo a Igreja Evangélica e Igreja Batista (HARTH, 2006).

A igualdade de gênero na política de saúde só pode ser alcançada quando as semelhanças e diferenças entre as necessidades de saúde dos homens e das mulheres são identificadas (DOYAL, 2000). Além disso, as políticas devem assegurar a igualdade de acesso a saúde, garantindo que as mulheres possam realizar seu pleno potencial para a saúde (DOYAL, 2000). Isto deve ser feito por meio de alocação igualitária dos recursos, que leve em conta as limitações que o atual modo de organização social coloca sobre cada gênero, em particular sobre as mulheres (UNTERHALTER, 2003; GREANY, 2008; DOYAL, 2000).

\section{Reforma do Setor da Saúde Como um Estudo de Caso}

A reforma do setor da saúde, entendida como um conjunto de processos incorrendo em mudanças na organização e funcionamento dos serviços de saúde, visa melhorar o desempenho do sistema existente e garantir respostas eficazes e igualitárias face aos desenvolvimentos futuros (KOIVUSALO \& OLLILA, 1997). A Reforma no setor da saúde engloba o contexto, o conteúdo e o processo pelos quais essas alterações são feitas (KOIVUSALO \& OLLILA, 1997; WALT \& GILSON, 1994). Portanto, as reformas do setor da saúde incorporam e reproduzem os valores já estabelecidos característicos dos setores e instituições de saúde, como o preconceito de gênero (STANDING, 1997; ELSON \& EVERS, 1998; GIDEON, 2000). Embora tenha havido a criação e implementação de políticas sensíveis ao gênero, reformas no setor de saúde ainda podem produzir resultados desiguais devido aos processos, práticas e ideologias existentes que resultam na exclusão de gênero ou na distribuição desigual de poder e status entre homens e mulheres (ELSON \& EVERS, 1998; GIDEON, 2000).

A exclusão social e a vulnerabilidade ocorrem em níveis diferentes, dependendo dos grupos e indivíduos (ABEL \& LLOYD-SHERLOCK, 2000). Há aqueles que estão excluídos de todos os serviços públicos, aqueles que são excluídos de um serviço, mas não de outro, aqueles que são excluídos dos serviços públicos de boa qualidade e aqueles que optaram por se auto excluir de serviços disponíveis ao público (ABEL \& LLOYD-SHERLOCK, 2000). Ocorrem variáveis ao longo do tempo em cada situação e até mesmo entre membros do mesmo grupo (ABEL \& LLOYDSHERLOCK, 2000). Todos os fatores acima influenciam nos distintos estados de saúde dos vários indivíduos e grupos de uma sociedade.

As mulheres tendem a ser expostas a uma maior vulnerabilidade social em fatores como a pobreza, a discriminação socioeconômica e política, podendo ter um padrão de saúde inferior ao dos homens (MURTHY \& BHATTACHARYA, 2010). A falta de cobertura adequada, ou nenhuma cobertura, para os serviços de saúde sexual e reprodutiva é um dos principais fatores que levam ao estado inferior de saúde das mulheres (MURTHY \& BHATTACHARYA, 2010). Os estudiosos do tema apontam a dualidade dos sistemas de saúde como causa, ainda que parcial, das desigualdades e ineficiências nos serviços de saúde materno (VICTORA et al. 2011):

Os sistemas de saúde têm um papel fundamental na garantia da gravidez e do parto seguros, porque há evidências claras de que (após ajustada a estatística ao nível de renda do país) a prestação e o acesso aos serviços de saúde materna, especialmente cuidados obstetrícios de emergência, estão associados a uma redução da mortalidade materna (RUIZ-RODRIGUEZ, 2009, p.150).

Uma tentativa de melhorar as condições existentes e as condições básicas da saúde reprodutiva - isto é, taxa de mortalidade materna e taxa de fecundidade adolescente - tem sido feita através da descentralização dos sistemas de saúde pública em todos os países da América Latina (MESA-LAGO, 2010). Isto ocorreu na década de 1980 e 1990 como parte da retórica reforma do setor da saúde, incluída nos programas de ajuste estrutural preconizados pelo Banco Mundial e o Fundo Monetário Internacional e apoiada pela conferência, basicamente feminista, das Nações Unidas sobre população e desenvolvimento, ocorrida no Cairo em 


\section{4 (MAYHEW, 2003).}

No período da reforma do setor da saúde entre os anos 1980 e 1990 políticas estatais de bem-estar orientadas por princípios de solidariedade foram substituídas por alternativas neoliberais, enfatizando interesses individuais (KOIVUSALO \& OLLILA, 1997). Na América Latina, isso significou o corte de gastos e a alteração no modo de prestação de serviços, incluindo parcerias público-privadas, maior participação do setor privado, bem como de organizações não governamentais (KOIVUSALO \& OLLILA, 1997; MESA-LAGO, 2008).

\section{Análise de Políticas e Reformas do Setor de Saúde}

Desde meados do século XIX na América Latina, a política social tem posto a maternidade como objeto de regulação pelo Estado, através dos esforços de movimentos de mulheres e movimentos higienistas que visam à modernização das práticas no modo de ter e criar filhos (MOLYNEUX, 2007). Essa política, logo a seguir, tornou-se mais claramente a resposta ou resultado do interesse de determinadas redes políticas, dominadas por instituições dirigidas por indivíduos que visavam apenas seu próprio interesse (LIPSKY, 1980). O complexo entrelaçamento de instituições e o uso de intenso bombardeamento de informações por grupos especialistas nessa tarefa tornou-se, portanto, crucial para a concepção e implementação dessas políticas (MACKINTOSH, 1992).

O campo da formulação de políticas de saúde não é diferente. A política de saúde é inerentemente sujeita ao conflito entre ações de solidariedade em oposição a ações que visam bem-estar individual, e é nessa situação que as escolhas são feitas (WALT \& GILSON, 1994). Dessa forma, o conteúdo da reforma vem a ser menos importante do que o contexto, o processo e os agentes envolvidos na reforma política (nos níveis internacional, nacional e regional) (WALT \& GILSON, 1994; WALT et al. 2008). Isto porque focar no conteúdo das políticas desvia a atenção necessária para compreender os processos que explicam porque as políticas aplicadas não dão os resultados desejados (WALT \& GILSON, 1994).

Valores e conceitos das causas e consequências da saúde e da doença orientaram todas as decisões políticas relacionadas com as reformas de saúde (WALT \& GILSON, 1994). E isto pode ser utilizado para influenciar a implementação da política, na prática ou como parte da retórica governamental (KOIVUSALO \& OLLILA, 1997). A diferença fundamental reside em qualificar a saúde como um fim em si mesmo - ou seja, intrinsecamente - ou como um meio - portanto como um valor relativo em comparação com outros objetivos mais valiosos (KOIVUSALO \& OLLILA, 1997).

É aqui que os direitos humanos e abordagens baseadas nesse conceito se tornam relevantes. Direitos humanos como valor intrínseco ou retórico significam a busca da saúde como um valor social fundamental indispensável para a igualdade e a aplicação da justiça (KOIVUSALO \& OLLILA, 1997; HUNT \& SHELDON 2010).

A razão por trás de uma reforma de saúde particular serão amplamente divulgadas pelos grupos políticos que participam de sua concepção e do processo de aplicação (MACKINTOSH, 1992). O sucesso ou fracasso de uma reforma dependerá não só da sua concepção, mas também dos atores e dos beneficiários que tomam parte em sua implementação (MAYHEW 2003; MCINTYRE \& KLUGMAN, 2003). Muitas vezes, estratégias de saúde idealizadas de forma internacional deixam de reconhecer que também existem enormes lacunas culturais, burocráticas, de uma estrutura política arcaica e de decisões que são tomadas nos níveis locais, no momento da implementação de políticas de reforma (WALT AND GILSON 1994; LIPSKY 1980).

Neste caso, para compreender os fatores que influenciam a eficácia ou ineficácia da mudança na política de saúde, é preciso entender o processo político (WALT \& GILSON 1994; MACKINTOSH 1992; WALT et al. 2008; GILSON \& THOMAS 2002). Isto significa dimensionar o ambiente político em termos internos, transnacionais (ou que cruza fronteiras de países vizinhos) e globais, com as múltiplas relações dos atores do processo (WALT et al. 2008). Isso politiza ou repolitiza as ações, tirando-as da esfera técnica e colocando-as no centro do discurso defendido pelas organizações de direitos humanos internacionais (CORNWALL \& NYAMU-MUSEMBI, 2004). Assim, destaca-se o crescimento da interdependência global e de estratégias internacionais e bilaterais em matéria de saúde, relativo a suas relações com a mudança da política nacional (WALT et al. 2008). Explora-se assim a relação entre a retórica política global, o poder e o grau de realização efetivo do discurso baseado em direitos (WALT et al. 2008). O uso de uma abordagem de análise política é dirigida para as questões de motivos e maneiras para que a mudança aconteça, bem como o modo tudo isso se conecta a reforma em si (GIDEON, 2000; GILSON \& THOMAS, 2002). 


\section{As Reformas do Setor de Saúde das décadas de 1980 e 1990}

A reforma dos sistemas públicos de saúde varia consideravelmente de país para país. Uma quantidade indeterminada de abordagens tem sido usada em diferentes contextos (MESA-LAGO, 2007). Estes vão desde reformas radicais até um espectro menor de mudanças, e podem ser agrupadas em mudanças no modo de financiamento, mudanças organizacionais, ou mudanças na estratégia política (MESA-LAGO, 2007). No entanto, pode-se dizer que a grande maioria dos países latino-americanos implementaram, em algum momento, durante as décadas de 1980 e 1990, algum tipo na reforma da saúde pública, razoavelmente abrangente, baseando-se em mudanças organizacionais (MESA-LAGO,2007).

Estas reformas podem ter promovido uma maior flexibilidade, eficiência e responsabilidade na utilização dos recursos, na participação e no envolvimento da comunidade (MESA-LAGO, 2007; 2008). Mas também podem ter levado à fragmentação do setor, a perda da liderança política, a confusão de responsabilidades e deterioração dos serviços (YAMIN, 2000; HOMEDES \& UGALDE, 2005; PRIBBLE, 2010).

A reforma no setor de saúde na América Latina produziu resultados diversos. As reformas podem ter sido positivas ou negativas para a saúde reprodutiva (MESA-LAGO, 2010). Mas dado que os analistas da reforma geral de saúde geralmente não compartilham os valores dos defensores da reforma da saúde reprodutiva, os efeitos negativos tendem a ser realçados (LUBBEN, 2002). A maioria dos países latino-americanos parecem ter tido, sobretudo, experiências negativas em termos de realização efetiva dos direitos reprodutivos e bem-estar (MEIER, 2010). Isso porque as reformas normalmente visam superar as estruturas de gestão que são deficitárias e a falta de incentivos de desempenho, deixando o status reprodutivo em si intocado (MESA-LAGO, 2010).

A falha em reduzir as mortes maternas no Brasil, apesar do grande crescimento econômico e as reformas de saúde extensas, é uma parte particularmente interessante deste quebra-cabeça. Durante os anos 1980 e 1990, alguns grupos de pressão social eram particularmente fortes: a Igreja Católica, a elite voltada para o mercado e ideologia neoliberal, o movimento de controle da população Malthusiano e Neo-Malthusiano (CORRÊA, 1993). Ao mesmo tempo, o direito de planejar a família e as condições reprodutivas eram fortes exigências do movimento feminista, já desde o final da década de 1970 (ALVAREZ, 1990). O movimento feminista conseguiu - por meio do ativismo de seus membros - influenciar a formulação da Constituição de 1988, para criar a instituição do Conselho Nacional dos Direitos da Mulher e o Programa de Atenção Integral à Saúde da Mulher (ALVAREZ, 1990). No entanto, o movimento feminista não conseguiu ganhar peso político suficiente para efetivamente influenciar o resultado da transição democrática, para moldar à sua maneira a implementação da reforma do setor da saúde na década de 1990 (ALVAREZ,1990).

\section{Redes Políticas e Descentralização de Serviços}

Reformas do setor de saúde são particularmente difíceis porque afetam um grande número de pessoas, seus subsídios diretos, a prestação de serviços é um processo complexo, a clientela é diversificada e o mercado de saúde é altamente imperfeito e desigual (MESA-LAGO, 2008). Em todos os casos, os que decidem a política influenciam de alguma forma a tomada das decisões quando aplicam suas escolhas políticas (HAAS, 1992). E, considerando os problemas políticos complexos gerados pela reforma do setor da saúde, aqueles que decidem tendem a optar por uma solução clara e já testada, muitas vezes a partir de modelos utilizados em países vizinhos (WEYLAND, 1996; EWIG, 2010).

Atores supranacionais e globais têm sido cada vez mais importantes nas mudanças de políticas (DIÁCONO et al, 1997). No caso da política de bemestar e estado social, instituições internacionais como a Organização Mundial do Trabalho, Organização Mundial da Saúde, Fundo Monetário Internacional e o Banco Mundial, por vezes se desviaram de seus objetivos iniciais, a fim de se engajar mais na reformulação das políticas e regras de cada país (DIÁCONO et al, 1997). Esta influência tornou-se particularmente aguda, de forma supranacional e nacional, nas décadas de 1980 e 1990, com os programas de ajuste estrutural voltados ao livremercado (DIÁCONO et al, 1997).

Ainda que uma abordagem, indubitavelmente, baseada em direitos date da criação da Organização Mundial da Saúde mesmo, esta definição ampla e positiva do direito à saúde foi deixada de lado durante uma mudança dramática de paradigma na Organização Mundial da Saúde, entre 1953 e 1973 (MESA-LAGO, 2008; MEIER 2010). Nessa época, a saúde global, foi tomada pelo jargão biomédico, bem como da busca por metas, como resultado dos avanços da ciência após a $2^{\mathrm{a}}$ Guerra Mundial (WALT \& GILSON 1994; MEIER, 2010). Esta mentalidade possivelmente veio em 
detrimento da meta do desenvolvimento e implementação do direito à saúde e a sua inclusão nas políticas de reforma do setor de saúde (KOIVUSALO \& OLLILA, 1997; WALT \& GILSON, 1994).

Dois relatórios, o World Development Report Investing in Health, de 1993, e o Averting Old-Age Crisis, de 1994, - que pediam a descentralização do sistema, com auto-suficiência e autonomia hospitalar foram particularmente importantes para esse fenômeno (MESA-LAGO, 2008). Na realidade, as características principais da reforma da saúde na América Latina nos anos 1990 foram a introdução de taxas para os serviços, um pacote básico de serviços de saúde e a descentralização da administração de secretarias municipais e postos de saúde locais (MESA-LAGO, 2008). Desta forma, a privatização e a descentralização foram introduzidos como uma condicional de contratos de empréstimo entre os países latino-americanos e o Banco Mundial e o Fundo Monetário Internacional (MESA-LAGO, 2008).

A política de descentralização foi disfarçada sob o princípio da transferência de poder dos burocratas distantes e ineficientes do governo central para estados e municípios como forma de democratizar os sistemas de saúde (HOMEDES \& UGALDE, 2005). Isso não apenas era muito controverso por causa da introdução dessas políticas sob regimes ditatoriais, mas também porque as avaliações dos esforços de descentralização na América Latina mostram que os 'objetivos políticos' raramente são cumpridos (HOMEDES \& UGALDE, 2005).

O Sistema de saúde no Brasil só foi descentralizado na década de 1980 como resultado de um amplo movimento por justiça social e econômica originada no grupo de medicina acadêmica intitulando Movimento pela Reforma Sanitária (COHN, 2008). O movimento pressionou por uma assistência universal preventiva, com acesso aos serviços básicos para todos, mas com especial atenção aos pobres (COHN, 2008).

A reforma do setor de saúde estava longe de ser o objetivo inicial do movimento (WEYLAND, 1996). Em seu início ele estava focado em demandas locais e distributivas, ao invés de grandes missões reformistas. Isso foi devido a fortes ligações com redes clientelistas, que representavam as empresas que visam o lucro, e que, portanto, resistiam a quaisquer alterações no status quo, bem como a visões divergentes sobre a questão do Ministério da Saúde e Instituto Nacional de Previdência Social (INPS, hoje INSS) (WEYLAND, 1996; COHN, 2008).

Diferente da maioria dos países da América Latina, as reformas do Brasil eram - pelo menos até meados dos anos 1990 - fortemente influenciadas pelo movimento médico esquerdista interno. No entanto, há relatos que apontam para a Declaração de Alma-Ata e as estratégias 'Saúde para Todos' da Organização Mundial de Saúde como uma fonte de inspiração e de guia ideológico (WEYLAND, 1996).

A reforma foi implementada em três fases: duas em meados da década de 1980 e uma no início da de 1990 . A primeira consistiu na descentralização da prestação de serviços do Ministério da Saúde do Instituto Nacional de Previdência Social para os estados e municípios, chamado desde 1983 de Ação Integrada pra Saúde (COHN, 2008). E a segunda fase ocorreu em 1987 e 1988 com a criação dos Sistemas de Saúde Unificados e descentralizados, que promoveram a descentralização administrativa através da transferência de pessoal e controle de instalações para secretarias estaduais e municipais de saúde (COHN, 2008). Posteriormente, como parte da transição para a democracia, a Constituição de 1988 incorporou os ideais da Carta Constitucional de Saúde e criou o Sistema Único de Saúde (COHN, 2008). O artigo 196 da Constituição de 1988 reconheceu a saúde como direito individual e coletivo ao acesso aos serviços de saúde (COHN 2008).

Em 1990, o Sistema Único de Saúde foi regulamentado pela Lei de Organização de Saúde composta pelas Leis Orgânicas 8.080/90 e 8.142/90 (LOBATO \& BURLANDY, 2000). A Lei de Organização subdivide o Sistema Único de Saúde em três setores: um setor público, um setor privado contratado financiado pelo setor público e um setor privado financiado pelos planos de saúde pagos (BUSS \& GADELHA, 1996). Ela integra uma série de provedores públicos, hospitais e centros de saúde primários no âmbito dos governos federal, estaduais e municipais e inclui também instituições privadas, tanto de caridade quanto que visam o lucro, remuneradas pelo governo (LOBATO \& BURLANDY, 2000; BUSS \& GADELHA, 1996).

Apesar desta estrutura tripla intrincada, o ideal de tornar políticas de saúde mais próximas das comunidades estava longe de ser eficaz. Na verdade, a Lei de Organização de Saúde parcialmente recentralizou várias medidas, dando de volta ao governo federal o direito de definir normas para a contratação de prestadores privados, e podendo novamente controlar a transferência das receitas federais a estados e municípios geridas pelo Sistema Único de Saúde (WEYLAND, 1996). Além disso, a luta pelo poder político, econômico e institucional dividiu após 1988 o movimento de saúde, comprometendo neste sentido os próprios princípios e razões que haviam unificado esses grupos anteriormente (BAPTISTA, 1996).

As novas formações políticas da ala esquerdista, 
que se diluiu, dividiu ainda mais o movimento de saúde reformista, deixando pouco espaço para iniciativas feministas (BAPTISTA, 1996). A única diretriz organizacional do Sistema Único de Saúde que foi amplamente apoiada, poupada da cruzada do neoliberalismo, foi a descentralização (BAPTISTA, 1996). Os problemas foram novamente agravados na década de 2000 com a oposição difusa da nova ala conservadora da Igreja Católica e Igrejas Evangélicas Cristãs, que se afastaram de enquadrar os debates públicos sobre questões de moralidade pessoal, indo para um discurso baseado nos direitos que anteriormente tinha sido feito apenas dentro da ala esquerda de oposição (MACAULAY, 2010).

Hoje o Sistema Único de Saúde caracteriza-se por uma descentralização que é extremamente dependente dos municípios e que é simultaneamente dependente do poder do governo federal sobre a transferência de recursos financeiros, bem como sobre as principais áreas de decisão política (BAPTISTA 1996).

Curiosamente, a não delegação de papéis significativos para os estados conduziu ao enfraquecimento do poder de barganha dos municípios, em relação ao governo federal, gerando conflitos políticos sobre as responsabilidades de cada um (BAPTISTA 1996). O sistema de saúde é definido como um setor altamente técnico e, portanto, regido por regras operacionais, deixando pouco espaço para os movimentos feministas, que pregam a saúde materna como um valor intrínseco (BAPTISTA 1996).

\section{Conclusão}

Nas décadas de 1980 e 1990, as reformas na saúde foram amplamente implementadas na América Latina. No entanto, os resultados delas têm sido conflitantes. As iniciativas brasileiras introduzidas no final da década de 1980 tinham como objetivo proporcionar o acesso integral e igualitário aos serviços de saúde, mas não puderam abarcar a enormidade da tarefa. As iniciativas de abordar apenas as questões dos recursos econômicos e técnicos, ignorando mais amplos contextos políticos e sociais relacionados a saúde são os fatores determinantes nesse resultado.

Enquanto os que tomam as decisões políticas forem pressionados para tomar parte e contribuir para a agenda dos governos focados apenas em questões financeiras e eficiência, as preocupações de saúde reprodutiva serão geralmente deixadas de lado, em favor das questões mais estabelecidas, tais como análise de custos e controle populacional. Neste sentido, a descentralização não garante um caráter democrático ao processo de tomada de decisão, e por esta razão marginaliza as redes e os atores que foram historicamente desfavorecidos. Isso pouco ou nada faz para melhorar o caso surpreendente de altas taxas de mortalidade materna no Brasil.

1 Tradução: Rafael Mendonça de Paula.

\section{Referências}

ABEL, Christopher; LLOYD-SHERLOCK, Peter. Health policy in Latin America: themes, trends and challenges". In: LLOYD-SHERLOCK, Peter (Ed.). Health Care Reform and Poverty in Latin America. London: Institute of Latin American Studies, 2000, p.143-162.

ALMEIDA, Celia; TRAVASSOS, Claudia; PORTO, Silvia; LABRA, Maria Eliana. Health Sector Reform in Brazil: A Case Study of Inequity. International Journal of Health Services, v. 30, n. 1, p. 129 - 162, 2000 .

ALKIRE, Sabina. Why the Capability Approach?" Journal of Human Development, v. 6, n. 1, p. 115 135, 2005.

ÁlVAREZ, Sônia. Engendering Democracy in Brazil: Women's Movements in Transition Politics. Princeton: Princeton University Press; 1990.

BAPTISTA, Tatiana Wargas de Faria. Caminhos e Percalços da Política de Saúde no Brasil: vinte anos de reforma sanitaria, Brasília, MS, Consultoria Projeto Nordeste II, 1996/1997, 71 p.

BUSS, Paulo; GADELHA, Paulo. Health care systems in transition: Brazil Part I: An outline of Brazil's health care system reforms. Journal of Public Health Medicine, v. 18, n. 3, p. 289 - 295, 1996.

COHN, Amélia. The Brazilian health reform: A victory over the neoliberal model. Social Medicine, v. 3, n. 2, p. $71-81,2008$.

CORNWALL, Andrea; MOLYNEUX, Maxine. The Politics of Rights: Dilemmas for Feminist Praxis: An Introduction. Third World Quarterly, v. 27, n. 7, p. 1175 - 1191, 2006.

CORNWALL, Andrea; NYAMU-MUSEMBI, Celestine. Putting the 'Rights-Based Approach to Development' into Perspective. Third World Quarterly, v. 25, n. 8, p. 1415 - 38, 2004. 
CORRÊA, Sônia. PAISM: uma história sem Fim. Revista Brasileira de Estudos Populacionais, v. 10, n. 1, p. 3 - 11, 1993.

CORRÊA, Sônia. Brazil: one of the abortion frontlines. Reproductive Health Matters, v. 18, n. 36, p. $111-117,2010$.

DEACON, Bob; HULSE, Michelle; STUBBS; Paul. Global Social Policy, International Organisations and the Future of Welfare. London: SAGE, 1997.

DOYAL, Leslie. What Makes Women Sick: Gender and the Political Economy of Health. London: Macmillan, 1995.

DOYAL, Leslie. Gender Equity in Health: Debater and Dilemas. Social Science \& Medicine, v. 51, p. 931 939, 2000.

ELSON, Diane; EVERS, Barbara. Sector Programme Support: The Health Sector - A Gender Aware Analysis. Manchester: University of Manchester, GENECON Unit, 1998.

EWIG, Christina. Second-Wave Neoliberalism: Gender, Race and Health Sector Reform in Peru. University Park: Penn State University Press, 2010.

GIDEON, Jasmine. The Politics of Health Reform in Chile: Gender and Participation in Primary Health Care Delivery. A thesis submitted to the University of Manchester for the degree of Doctor of Philosophy in the Faculty of Economic and Social Sciences; 2000.

GILSON, Lucy; THOMAS, Stephen. Informing Policy and Practice: Developing a Policy Analysis Framework for Evaluation of Health System Change. In: Project Analysing the processes of health financing reform in South African and Zambia (the SAZA project), University of Cape Town and University of Witwatersrand; 2002.

GREANY, Katie. Rhetoric versus reality: exploring the rights-based approach to girls education in rural Niger. Compare: A Journal of Comparative and International Education, v. 38, n. 5, p. 555 - 568, 2008 .

HAAS, Peter. Introduction: Epistemic Communities and International Policy Coordinations. International Organizations, v. 46, n. 1, p. 1 - 35, 1992.

HARTH, Elfriede. The impact of religion on sexual and reproductive health and rights in Latin America. Catholics for Choice - Presentation to the European Parliament, p. $1-6,2006$.

HOGAN, Margaret; FOREMAN, Kyle; NAGHAVI, Mohsen (et al). Maternal mortality for 181 countries, 1980 - 2008: a systematic analysis of progress towards Millennium Development Goal 5. Lancet, v. 375, n. 9726, p. 1609 - 1623, 2010.

HOMEDES, Núria; UGALDE, Antonio. Why neoliberal reforms have failed in Latin America? Health Policy, v. 71, p. 83 - 96, 2005.

HUNT, Paul; LEADER, Sheldon. Developing and applying the right to the highest attainable standard of health: The role of the UN Special Rapporteur (20022008). In: HARRINGTON, John; STUTTAFORD, Maria. Global health and human rights: legal and philosophical perspectives. New York: Routledge, 2010 , p. $28-61$.

KOIVUSALO, Meri; OLlILA, Eeva. Making a healthy world: agencies, actors and policies in international health. London: Zed Books, 1997.

LIPSKY, Michael. Street Level Bureaucracy. New York: MacMillan, 1980.

LOBATO, Lenaura; BURLANDY, Luciene. The Context and Process of Health Reform in Brazil. In: FLEURY, Sônia; BELMARTINO, Susana; BARIS, Enis. Reshaping Health Care in Latin America: A Comparative Analysis of Health Sector Reform in Argentina, Brazil, and Mexico. Ottawa: International Development Research Council Press, 2000, p. $79-$ 102.

LUBBEN, Marianne; MAYHEW, Susannah; COLLINS, Charles; GREEN, Andrew. Reproductive Health and Health Sector Reform in Developing Countries: Establishing a Framework for Dialogue. Bulletin of the World Health Organization, v. 80, n. 8, p. $667-674,2002$.

MACAULAY, Fiona. Trickling Up, Down, and Sideways: Gender Policy and Political Opportunity in Brazil. In: MAIER, Elizabeth; LEBON, Natalie (eds.). Women's Activism in Latin America and the Caribbean: Engendering Social Justice, Democratizing Citizenship. New Jersey: Rutgers University Press, 2010, p. $273-290$.

MACKINTOSH, Maureen. Questioning the state. In: 
WUYTS, Marc; MACKINTOSH, Maureen; HEWITT, Tom (eds.). Development Policy and Public Action. Milton Keynes: Open University Press, 1992, p. 61 80 .

MCINTYRE, Di; KLUGMAN, Barbara. The Human Face of Decentralisation and Integration of Health Services: Experience from South Africa. Reproductive Health Matters, v. 11, n. 21, p. 108 - 119, 2003.

MEIER, Benjamin Mason. Global Health Governance and the Contentious Politics of Human Rights: Mainstreaming the Right to Health for Public Health Advancement. Stanford Journal of International Law, v. 46, n. 1, p. 1 - 50, 2010.

MESA-LAGO, Carmelo. Social Security in Latin America Pension and Health Care Reforms in the Last Quarter Century. Latin American Research Review, v. 42, n. 2, p. $181-201,2007$.

MESA-LAGO, Carmelo. Reassembling Social Security: A Survey of Pensions and Health Care Reforms in Latin America. Oxford: University of Oxford Press, 2008.

MESA-LAGO, Carmelo. World Crisis Effects on Social Security in Latin America and the Caribbean: Lessons and Policies. London: Institute for the Study of the Americas, 2010.

MOLYNEUX, Maxine. Change and Continuity in Social Protection in Latin America: Mothers at the Service of the State? In: Gender and Development Programme, Paper Number 1, Geneva: UNRISD, 2007.

MAYHEW, Susannah. The Impact of Decentralisation on Sexual and Reproductive Health Services in Ghana. Reproductive Health Matters, v. 11, n. 21, p. 74 - 87, 2003.

MURTHY, Padmini; BHATTACHARYA, Dhrubajyoti. Human Rights and Women's Health. In: BERACOCHEA, Elvira; WEINTEN, Corey; EVANS, Dabney (eds.). Rights-Based Approaches to Public Health. London: Springer, 2010, p. $169-182$.

NUSSBAUM, Martha. Women and Equality: The capabilities approach. In: LOUTFI, Martha Loutfi (Ed.). What is Equality and How We Get There? Women, Gender and Work. Geneva: International Labour Organization, 2001, p. 45 - 68.
PRIBBLE, Jennifer. Universalizing Healthcare Policy in Chile and Uruguay. Paper Prepared for delivery at Annual Meeting of the American Political Science Association, Washington, DC: 2010.

RUIZ-RODRÍGUEZ， Myriam; WIRTZ， Veronika; NIGENDA, Gustavo. Organizational elements of health service related to a reduction in maternal mortality: The cases of Chile and Colombia. Health Policy, v. 90, p. 149 - 155, 2009.

SEN, Amartya. The Possibility of Social Choice. American Economic Review, v. 89, n. 3, p. 349 - 378, 2002.

STANDING, Hilary. Gender and Equity in Health Sector Reform Programmes: a Review. Health Policy and Planning, v. 12, n. 1, p. 1 - 18, 1997.

UNDP. Human Development Report 2010 - The Wealth of Nations: Pathways to Human Development. New York: United Nations Development Fund, 2010.

UNTERHALTER, Elaine. The capabilities approach and gendered education: An examination of South African complexities. Theory and Research in Education, v. 1, n. 1, p. 7 - 22, 2003.

UNTERHALTER, Elaine. Partnership, participation and power for gender equality in education. In: Situation Analysis prepared for the UNGEI E4 Conference, January 2010.

VAUGHAN, Rosie Peppin. Girls' and women's education within Unesco and the World Bank, 19452000. Compare: A Journal of Comparative and International Education, v. 40, n. 4, p. 405 - 423, 2010.

VICTORA, Cesar; AQUINO, Estela; LEAL, Maria do Carmo et al. Maternal and child health in Brazil: progress and challenges. Lancet, v. 377, p. 1863 1876,2011

WALT, Gill; GILSON, Lucy. Reforming the health sector in developing countries: the central role of policy analysis. Health policy and planning, v. 9, n. 4, p. 353 - 370, 1994.

WALT, Gill; SHIFFMAN, Jeremy; SCHNEIDER, Helen et al. 'Doing' health policy analysis: methodological and conceptual reflections and challenges. Health Policy Plan, v. 23, n. 5, p. 308 - 17, 
Colocando a Saúde Materna no Centro das Políticas de Saúde Pública

2008.

WEYLAND, Kurt. Democracy Without Equity: Failures of Reform in Brazil. Pittsburgh: University of Pittsburgh Press, 1996.

WHO. Maternal mortality in 2000: estimates developed by WHO, UNICEF and UNFPA. Geneva: World Health Organization; 2004.

WHO, UNICEF, UNFPA et al. Trends in Maternal Mortality: 1990 to 2008 - Estimates by WHO, UNICEF, UNFPA and the World Bank. Geneva: World Health Organization, 2010.

YAMIN, Alicia Ely. Protecting and Promoting the Right to Health in Latin America: Selected Experiences from the Field. Health and Human Rights, v. 5, n. 1, p. 116 - 148, 2000. 